\title{
Efecto del sustrato en la intensidad del asentamiento de larvas de Argopecten purpuratus Lamarck, 1819 (Bivalvia, Pectinidae) en ambiente controlado
}

\author{
Lucio Encomendero ${ }^{1} \&$ Enrique Duprée \\ ${ }^{1}$ Universidad Nacional del Santa. Apartado 10, Chimbote-Perú \\ ${ }^{2}$ Universidad Católica del Norte, Sede Coquimbo. Casilla 117, Coquimbo, Chile \\ E-mail: edupre@ucn.cl
}

\begin{abstract}
RESUMEN. Se estudió el efecto de diferentes tipos de sustrato y del biofilm formado sobre ellos, en la intensidad del asentamiento larval del ostión del norte Argopecten purpuratus. Se postula la hipótesis que sustratos de diferente naturaleza permiten la formación de diferentes biofilm a través del tiempo, los cuales originan distintas intensidades de asentamiento larval sobre ellos. Sustratos de netlón, malla mosquitera, concha de ostión y asbesto-cemento se sometieron a la formación de biofilm por períodos de 6,12 y 18 días con flujo de agua filtrada a $50 \mu \mathrm{m}$. A cada sustrato con biofilm de diferentes edades (6,12 y 18 días) o sin él, contenidos en un recipiente con $300 \mathrm{~mL}$ de agua de mar filtrada, se le agregaron 200-300 larvas competentes. Después de 72 h en condiciones de laboratorio $\left(15,3-18,6^{\circ} \mathrm{C}\right)$, se contó el número de larvas asentadas. Se determinó que el tipo de sustrato influye en la intensidad del asentamiento larval del ostión. Los mayores asentamientos se obtuvieron sobre asbesto-cemento (29,6-62,3\%); seguidos por malla mosquitera (17,1-21,2\%) y concha de ostión (13-30\%). Los niveles más bajos se obtuvieron sobre el netlón (4,315,2\%). También se observó una relación directa entre el porcentaje de asentamiento larval y la edad del biofilm excepto en asbesto-cemento. El tipo de sustrato y la edad del biofilm mostraron un efecto sinérgico sobre la intensidad del asentamiento larval.
\end{abstract}

Palabras claves: asentamiento, larvas, sustrato, biofilm, ostión.

\section{Effect of different susbstrates on the larval settlement of the scallop Argopecten purpuratus Lamarck, 1819 (Bivalvia, Pectinidae) in laboratory condition}

\begin{abstract}
The effects of different types of substrate and of primary biofilm formed on them in relation to their capacity to attract settlement of competent larvae of the northern scallop Argopecten purpuratus, was studied. The hypothesis that different substrates permit the formation of different biofilm over time, which affect the intensity of scallop larval settlement on each different substrate, was tested. Substrates including Netlon @, fly screen, scallop shell, and asbestos-cement were incubated in $50 \mu \mathrm{m}$-filtered flowing sea water to allow formation of primary biofilm for periods of 6, 12, and 18 days. Each substrate, fouled for the time periods listed, was exposed to 200-300 competent scallop larvae in test volumes of $300 \mathrm{~mL}$ filtered seawater for periods of $72 \mathrm{~h}$ at ambient temperature $\left(15.3-18.6^{\circ} \mathrm{C}\right)$, after which the numbers of larvae settled on each substrate were determined. The results showed that the type of substrate influenced the intensity of scallop larval settlement. The highest settlement occurred on the asbestos-cement (29.6-62.3\%); intermediate levels of settlement were observed on the fly screen (17.1-21.2\%) and scallop shell (13.0$30.0 \%$ ), and the lowest levels occurred on the Netlon (4.3-15.2\%). A direct relationship was also observed between the percentage of larval settlement and the age of the biofilm, except for asbestos-cement. The type of substrate and age of the biofilm showed a synergistic effect on the intensity of larval settlement.
\end{abstract}

Key words: larval settlement, substrate, biofilm, scallop.

\section{INTRODUCCIÓN}

Una de las etapas más críticas del ciclo de vida de los invertebrados bentónicos es el asentamiento. Esta etapa que comprende tres fases: conducta de asentamiento, fijación y metamorfosis (Bonar et al.,
1990), puede prolongarse o no realizarse si las larvas competentes no encuentran un sustrato y estímulo adecuado para el asentamiento (Crisp, 1974; Bayne, 1975; Sastry, 1979; Tapia et al., 1993). Los factores ambientales que influyen en el asentamiento larval de diferentes especies son: físicos (flujo, pre- 
sión, temperatura, luz), biológicos (bacterias, microflora, microfauna) y físico-químicos del sustrato basal (tipo de material, porosidad, textura, color, energía libre superficial) (Hadfield, 1986; Jackson, 1986; Yool et al., 1986; Bonar et al., 1990; Pawlik \& Hadfield, 1990; Jensen et al., 1990; Bech, 1992; Zimmer-Faust \& Tamburri, 1994).

Diversas investigaciones muestran que las larvas de moluscos se asientan en respuesta a un tipo específico de película biológica o biofilm formado sobre un sustrato (Fitt et al., 1990; Tamburri et al., 1992; Wainman et al., 1996), en cambio otras exhiben una variedad de respuestas frente a la presencia o ausencia del biofilm (Maki et al., 1989; 1992). Maki et al. (1992) demostraron que películas monoespecíficas de bacterias pueden estimular, inhibir la fijación o no tener efecto sobre la fijación de las larvas cypris de Balanus amphitrites, señalando que la edad del biofilm determina el aumento o disminución del asentamiento. Wolfaart et al. (1994) señalan que los mayores porcentajes de asentamientos inducidos por una película biológica se producen después de su estabilización, que ocurre entre los 14 y 21 días de iniciada su formación y que su influencia es variable en los días previos a la estabilización.

Los cultivos del ostión del norte, Argopecten purpuratus (Lamarck, 1819), en Perú y Chile, dependen del éxito del asentamiento larval durante la captación de semillas que se realiza en el mar, al igual que A. irradians (Ambrose et al., 1992). En estas condiciones el asentamiento o captación de semilla es aleatoria y condicionada por factores ambientales que afectan la producción de larvas, por lo cual es necesario producir semillas en laboratorios de ambiente controlado o hatcheries. En este proceso, el asentamiento larval es fundamental para obtener altos rendimientos en la producción de semilla.

El logro de altos asentamientos de larvas de bivalvos requiere de sustratos adecuados que permitan la formación de un adecuado biofilm sobre éste (Brand \& Hoogesteger, 1980). Esto significa identificar la estructura físico-química del sustrato que permite la fijación de microorganismos (biofilm), que favorezcan el asentamiento de larvas. El conocimiento de estas características, permitirá elegir otros tipos de materiales de menor costo que pudieran ser utilizados en el diseño de nuevos colectores de alto rendimiento en el asentamiento larvas tanto en el mar como en laboratorio.
Como primera etapa para conocer las características físicas y químicas de los sustratos que favorecen el asentamiento larval, mediante la adhesión de microorganismos que forman un biofilm específico, en el presente trabajo se determina la intensidad del asentamiento larval de A. purpuratus, inducida por una película biológica instalada en diferentes tipos de sustratos.

\section{MATERIALES Y MÉTODOS}

Se realizaron 16 experimentos por duplicado y con cuatro réplicas cada uno, obteniéndose un total de 128 observaciones. Se realizaron combinaciones de cuatro sustratos diferentes con biofilm de cuatro edades diferentes. En cada experimento se usaron 200-300 larvas competentes de A. purpuratus.

La selección de larvas competentes se realizó tamizando larvas provenientes de cultivos comerciales cuya edad varió entre 16 y 22 días. Se utilizó un tamiz de $150 \mathrm{~mm}$ para obtener larvas de 225-280 $\mu \mathrm{m}$ de longitud ántero-posterior y se acondicionaron por $24 \mathrm{~h}$ en recipientes con 3 litros con agua de mar microfiltrada $(0,22 \mu \mathrm{m})$ y esterilizada (AMFE), aireación constante y alimentación con Isochrysis galbana y Chaetoceros gracilis en proporción 1:1 a una concentración final de $3-4 \cdot 10^{4}$ cél $\cdot \mathrm{mL}^{-1}$, luego se lavaron con AMFE y se depositaron en un vaso de precipitación con $200 \mathrm{~mL}$ de AMFE. Con pipetas Pasteur se tomaron pequeñas alícuotas con larvas y se depositaron en placas Petri y se seleccionaron, bajo un microscopio estereoscópico, aquellas que mostraban características de larvas competentes sobre la base de los cambios conductuales de pre-asentamiento descritos por Tapia et al. (1993). Las características conductuales se complementaron con características morfológicas, como presencia de la mancha ocular, tamaño de la larva y presencia y desarrollo del pie (Tamburri et al., 1992). Posteriormente se transfirieron entre 200 y 300 larvas competentes a cada uno de los vasos de precipitado de $100 \mathrm{~mL}$, en cuyo fondo se ubicaron los diferentes sustratos con sus respectivos biofilm.

Como sustrato se utilizaron trozos de $16 \mathrm{~cm}^{2}(4 \mathrm{x} 4$ $\mathrm{cm})$ de los siguientes materiales: concha de ostión A. purpuratus (origen orgánico), asbesto-cemento (origen inorgánico), malla plástica con poros de 0,3 cm de diámetro (malla mosquitera) y malla de netlón (ambas mallas de materiales sintéticos). Cada sustrato se esterilizó usando hipoclorito de sodio comercial al $50 \%$ por 24 h. Después de lavados y 
biologizados, se sometieron a la formación de una película biológica natural de microorganismos (biofilm), manteniendo los sustratos en posición vertical y ordenados al azar en el fondo de un estanque de $500 \mathrm{~L}$ con flujo constante $\left(5,1 \mathrm{~L} \cdot \mathrm{min}^{-1}\right)$ de agua de mar filtrada a $50 \mathrm{~mm}$ a $15-17^{\circ} \mathrm{C}$ por tres periodos diferentes. Se formaron biofilm de 6, 12 y 18 días. Como controles se utilizaron los cuatro tipos de sustratos lavados en igual forma que los anteriores pero sin ser sometidos a la formación de película biológica. Todos los sustratos se usaron una sola una vez.

Posteriormente, se ubicaron los cuatro sustratos diferentes con biofilm de la misma edad en el fondo de un mismo recipiente y se agregaron 200-300 larvas competentes. Se utilizaron recipientes de plásticos transparentes de $18 \mathrm{~cm}$ de diámetro y $5 \mathrm{~cm}$ de altura, conteniendo $300 \mathrm{~mL}$ de agua de mar filtrada a 0,2 $\mu \mathrm{m}$ y esterilizada en autoclave. Luego se agregaron $30 \mathrm{~mL}$ de microalgas I. galbana para dar una concentración final de 3,6-5,5 $10^{4} \mathrm{cél} \cdot \mathrm{mL}^{-1}$. Tanto los experimentos como sus réplicas se realizaron en duplicado y a una temperatura de $15,3-18,6^{\circ} \mathrm{C}$.

Después de 72 h, mediante una lupa binocular se determinó el número de larvas asentadas en cada uno de los sustratos. Los resultados se expresaron tanto en larvas totales por cada sustrato como larvas $\cdot \mathrm{cm}^{-2}$ de sustrato. Se consideró como larva asentada, aquella que permaneció adherida al sustrato después del traslado del sustrato desde el recipiente experimental a una placa Petri para el recuento.

Para la determinación del área real de cada sustrato, se usaron diferentes cálculos. Para el ostión se cubrió con papel de aluminio toda el área haciendo que el papel tomara la forma del relieve de la concha, luego se recortó el papel siguiendo los bordes del sustrato y se estiró y aplanó la porción de papel usada para obtener la dimensión real de cada lado de la porción de sustrato. Para las mallas mosquitera y netlón, asumiendo que ambas están constituidas por filamentos cilíndricos en toda su extensión, se midieron los diámetros de 10 filamentos usando un ocular micrométrico y se calculó el diámetro promedio. Después se midió la longitud total de los filamentos que constituyeron cada ma1la. Con el diámetro y longitud total de los filamentos se aplicó la fórmula del área del cilindro.

Se determinó la homogeneidad de las varianzas mediante prueba de Barttlet y luego un análisis de varianza de dos vías $(a=0,05)$. Después, se aplicó el test de Tukey para determinar diferencias significativas entre las medias de asentamientos en cada sustrato. Se compararon, tanto los porcentajes totales de asentamientos por cada sustrato previa transformación de los porcentajes a arcsen, como el número de larvas asentadas por unidad de área.

\section{RESULTADOS}

\section{Asentamiento larval con relación al tipo de sustrato}

Los mayores porcentajes de larvas asentadas se produjeron sobre asbesto-cemento, variando entre $62,3 \%$ sin biofilm y $29,6 \%$ con biofilm de 18 días, observándose una disminución de los asentamientos al aumentar la edad del biofilm (Fig. 1). Los menores porcentajes se obtuvieron sobre netlón, que variaron entre $5 \%$ sin biofilm y $13,7 \%$ con biofilm de 18 días. Se observó un aumento de asentamientos a medida que aumentó la edad del biofilm. Intensidades intermedias se obtuvieron sobre la concha de ostión y malla mosquitera, existiendo en ambos sustratos una relación directa entre asentamientos y edad del biofilm. En concha de ostión, los asentamientos fueron $16 \%$ sin biofilm, $16,9 \%$ y $36,3 \%$ con biofilm de 6 y 18 días respectivamente, y en malla mosquitera $15,5 \%$ sin biofilm, $15,1 \%$ y $20,7 \%$ con biofilm de 6 y 18 días respectivamente (Fig. 1). El asbesto-cemento presentó asentamientos significativamente mayores respecto a los otros tres sustratos. Pero los asentamientos sobre concha de ostión, malla mosquitera y netlón, no fueron significativamente diferentes entre ellos (Fig. 2). Los porcentajes promedios de las intensidades de asentamiento fueron entre 0 y 18 días sobre malla mosquitera y sobre concha de ostión no fueron significativamente diferentes entre sí, pero sí con respecto al asbesto-cemento y netlón $(\mathrm{P}<0,005)$ (Fig. 3).

La intensidad de asentamiento por unidad de área fue significativamente diferente sólo entre asbestocemento $\left(0,7\right.$ larvas $\left.\cdot \mathrm{cm}^{-2}\right)$ y cada uno de los otros tres sustratos: netlón $\left(0,2\right.$ larvas $\left.\cdot \mathrm{cm}^{-2}\right)$, concha de ostión $\left(0,3\right.$ larvas $\left.\cdot \mathrm{cm}^{-2}\right)$ y malla mosquitera $(0,4$ larvas. $\mathrm{cm}^{-2}$ ) (Fig. 3).

\section{Asentamiento en relación al biofilm}

El análisis de varianza de doble vía no mostró efectos significativos en la intensidad de asentamiento de las larvas en relación con la edad del biofilm. Sin embargo, se observó una disminución de los 


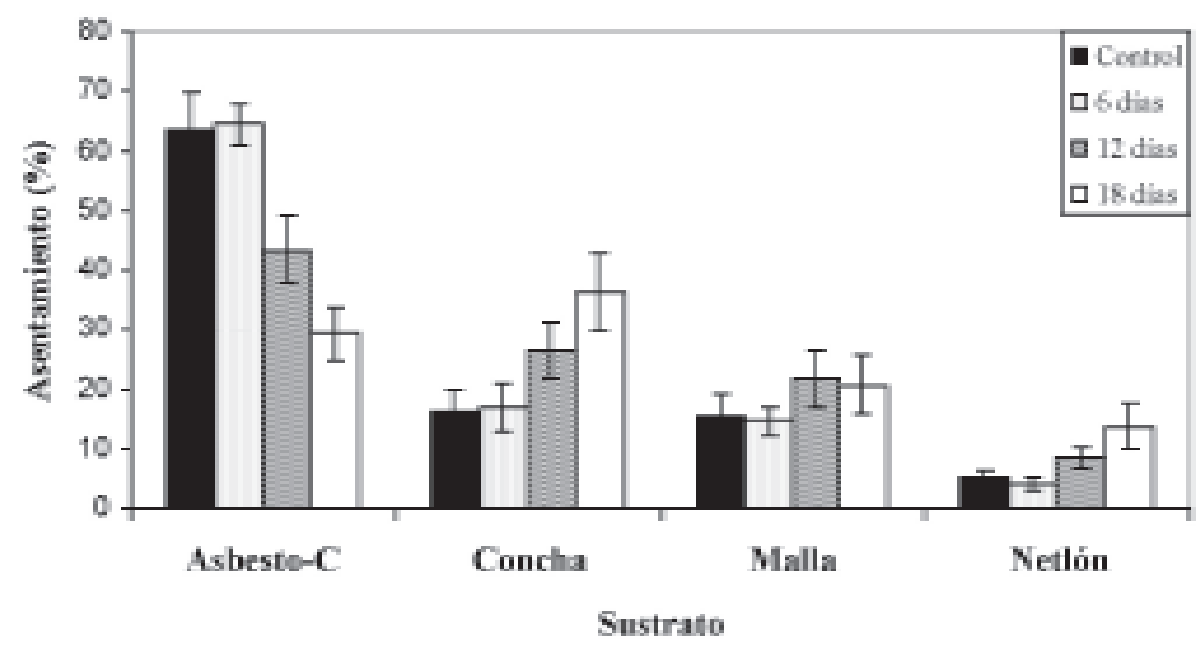

Figura 1. Porcentajes de asentamiento larval de Argopecten purpuratus sobre diferentes sustratos con biofilm de diferentes edades. Asbesto-C: asbesto-cemento, Malla: malla mosquitera, Concha: concha de ostión.

Figure 1. Percentages of larval settlement of Argopecten purpuratus on different substrates covered with different age biofilm. Asbesto-C: asbestos-cement, Malla: fly net, Concha: scallop shell.

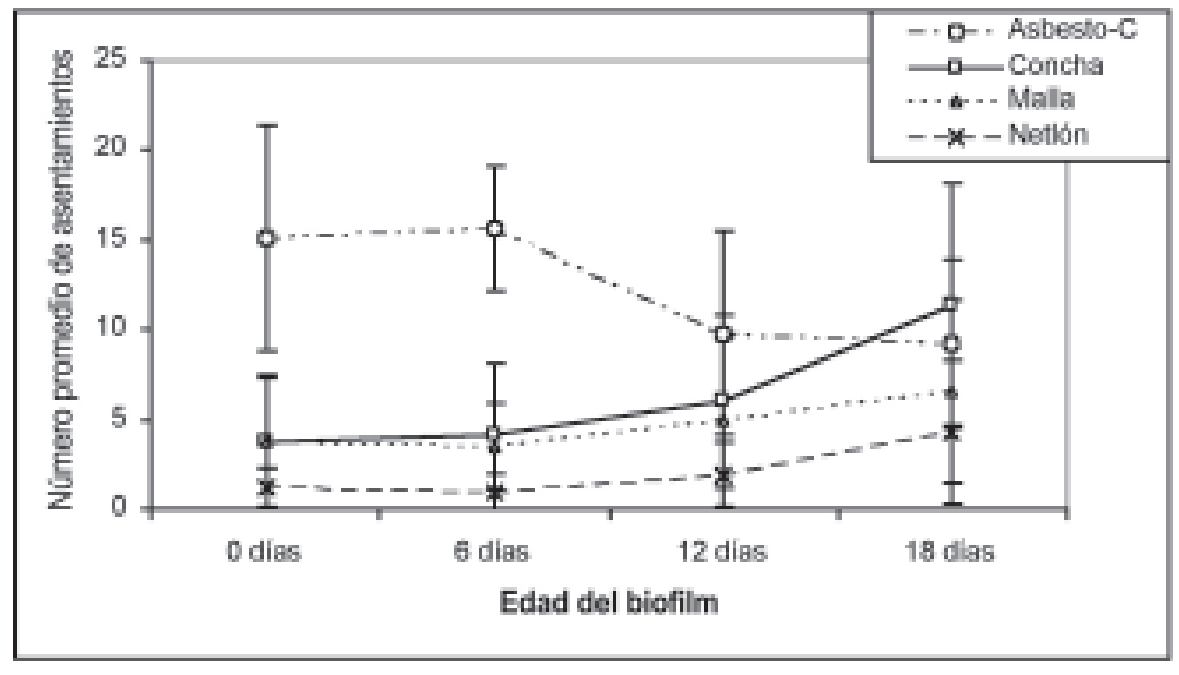

Figura 2. Número de asentamientos larvales promedios de Argopecten purpuratus sobre biofilm de diferentes edades en diferentes sustratos. Asbesto-C: asbesto-cemento, Malla: malla mosquitera, Concha: concha de ostión.

Figure 2. Average number of settled larvae of Argopecten purpuratus on different biofilm of different substrata. Asbesto-C: asbestos-cement, Malla: fly net, Concha: scallop shell. 


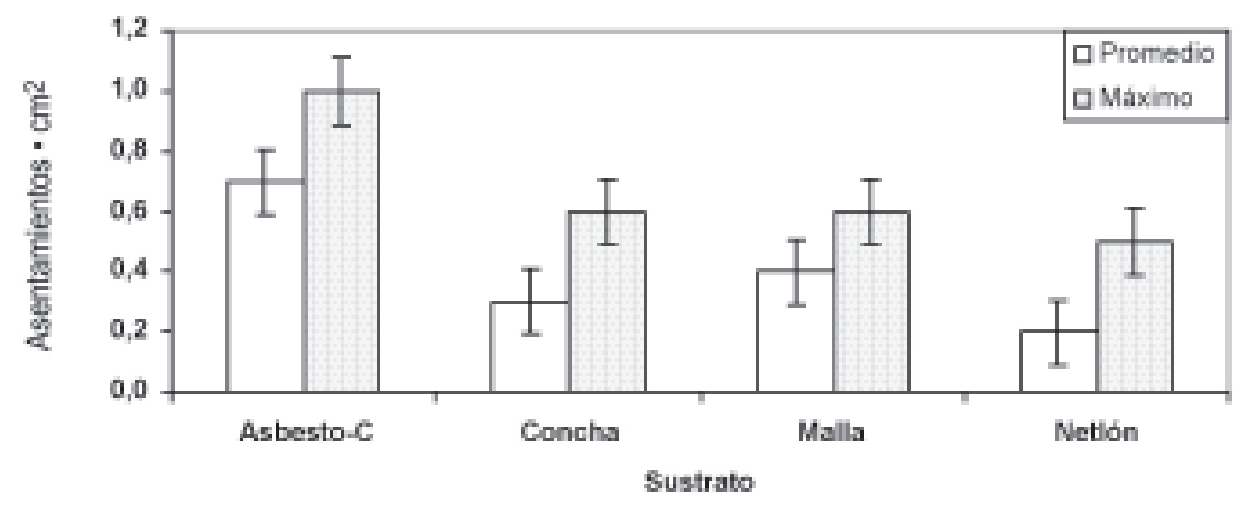

Figura 3. Número de asentamientos promedio y máximo por $\mathrm{cm}^{2}$ de diferentes sustratos, de Argopecten purpuratus entre los 0 y 18 días. Asbesto-C: asbesto-cemento, Malla: malla mosquitera, Concha: concha de ostión.

Figure 3. Average and maximun setllement of scallop larvae per $\mathrm{cm}^{2}$ of Argopecten purpuratus on different substrates from 0 to 18 days. Asbesto-C: asbestos-cement, Malla: fly net, Concha: scallop shell.

asentamientos sobre asbesto-cemento en relación con la edad del biofilm, siendo $64,5 \%$ a los 6 días, $43,4 \%$ a los 12 días y 29,3\% el día 18 . En cambio, en los otros tres sustratos se observó un aumento en el número de larvas asentadas entre el día 0 y 18 (Fig. 2). Aun cuando se observó una disminución del porcentaje de larvas que se asientan en el asbesto-cemento a medida que aumentó la edad del biofilm, estos porcentajes siempre fueron superiores a los otros sustratos con biofilm de cualquiera edad, excepto en concha de ostión con biofilm de 18 días que superó el asentamiento de 18 días de asbesto-cemento (Fig. 1). Lo mismo ocurrió al comparar los asentamientos por área de sustrato.

\section{DISCUSIÓN}

Los resultados obtenidos muestran que la intensidad del asentamiento larval de A. purpuratus varía de un sustrato a otro. El sustrato de asbesto-cemento presentó las más altas intensidades de asentamiento, el netlón las más bajas, y la concha de ostión y malla mosquitera niveles intermedios de intensidad. El mayor asentamiento sobre asbesto-cemento se debería al contenido de hidróxido de calcio, como lo determinó Anderson (1996) en Saccostrea commercialis, quien sostiene que el hidróxido de calcio como señal química, tiene efecto positivo sobre el asentamiento y que la larva responde a esa señal tanto en laboratorio como en ambiente natural. Si esta señal química está actuando sobre el asentamiento, sería una señal que actúa localmente y no difusible al medio, pues al colocar muestras de asbesto-cemento junto a los otros tres sustratos en un mismo recipiente experimental, sólo se obtuvieron altos porcentajes de asentamiento sobre el asbesto-cemento. Si la señal química fuera difusible y no influyera la estructura física de los materiales que constituyen el sustrato, los asentamientos habrían sido similares en los cuatro sustratos, lo cual no ocurrió.

Estas diferencias de asentamiento se deberían también a la gran heterogeneidad superficial del asbesto-cemento. Al respecto Bourget et al. (1994) señalan que las superficies ásperas tienen mayor área superficial y mayor diversidad de microambientes que las superficies lisas y en consecuencia, es mayor la posibilidad que se manifiesten diferentes mecanismos de sucesión de microcomunidades residentes que favorezcan los mecanismos de asentamiento de larvas. Sin embargo, Jacobi \& Lagevin (1996) sostienen que el efecto del sustrato original es efímero, y en definitiva, es la comunidad sésil local que se asienta sobre los sustratos, la que rige el asentamiento de la fauna móvil. Esta última sugerencia es posible que se esté dando en nuestros experimentos, ya que se observó una tendencia a disminuir los porcentajes de asentamientos sobre el asbesto-cemento y un aumento sobre los otros tres sustratos (concha, malla y netlón) cuando la edad de los biolfim aumentó.

Por lo tanto para el asbesto-cemento, se sugiere que al inicio, los asentamientos estarían favorecidos por una señal química originada en su naturaleza química y posteriormente, esta señal sería enmascarada por el biofilm que se va asentando sobre el 
sustrato, influyendo en mayor medida en este periodo el tipo de biofilm asentado sobre el sustrato.

Todd \& Keough (1994), sostienen que una de las causas de variabilidad temporal en el asentamiento larval es la heterogeneidad del hábitat, el cual está influenciado por la naturaleza física y química del sustrato. Además, Anderson \& Underwood (1994) señalan que las larvas prefieren asentarse en materiales que son fibrosos o porosos, al igual que lo determinado en el presente estudio.

Al comparar los asentamientos sobre sustratos sin biofilm, se observó que la malla mosquitera presentó mayores porcentajes de asentamientos larvales respecto al netlón (ambos de material sintético), lo cual podría deberse a que la malla está constituida por filamentos de menor diámetro $(0,25 \mathrm{~mm})$, que el netlón (0,8 mm), ya que Harvey et al. (1995) señalan que existe una relación inversa entre el diámetro de los filamentos colectores y el asentamiento larval. Resultados similares obtuvieron Pearce \& Bourget (1996) en filtros de poliester para acuarios que no presentan biofilm, donde se observó que este sustrato captó mayor número de larvas que otros sustratos con filamentos de mayor diámetro. Sin embargo, Miron et al. (1995) observaron que el diámetro del filamento no tiene un rol importante en el asentamiento de Placopecten magellanicus. Por otro lado, la estructura de los filamentos de malla mosquitera tiene poros de $0,1-0,5 \mathrm{~mm}$, lo cual permite ofrecer a los microorganismos formadores de biofilm, una mayor diversidad de micrositios para su adherencia y permitir una mayor diversidad de microorganismos sobre el biofilm que favorecen el asentamiento.

El mayor porcentaje de asentamientos sobre concha de ostión respecto a ambas mallas (mosquitera y netrón), se explicaría por la heterogeneidad de su superficie que ofrece mayor número de microambientes, que influirían sobre la densidad y composición taxonómica de la comunidad colonizadora (Prieur et al., 1993), y así actuar sobre la conducta de los colonizadores como larvas y otros organismos que realizan una activa selección de los microambientes de residencia (Harvey et al., 1995).

Las películas jóvenes no atraen tanto a las larvas como las películas viejas (Todd \& Keough, 1994). Anderson \& Underwood (1994) señalan que los biofilm de mayor edad están constituidos por microalgas y bacterias. Estas últimas son importantes para el asentamiento de las larvas, pues se fijan a los sustratos mediante exopolímeros (glicoproteínas, polisacáridos, glicolípidos, D-glucosa) (Marshall, 1989), produciendo una cubierta aniónica que despolarizaría las membranas plasmáticas de las células receptoras de estímulos de las larvas para iniciar la metamorfosis (Weiner et al., 1993).

El sinergismo del sustrato y biofilm asentado, sobre el observado en este estudio, indicaría que la naturaleza de la superficie del sustrato influye en la velocidad de conformación o composición específica del biofilm y que a su vez, la película influiría en el efecto de los sustratos en la intensidad del asentamiento larval, ya que las larvas usan diatomeas, bacterias, exopolisacáridos o detritos, como señales para asentarse (Weiner et al., 1993; Bourget et al., 1994).

En las predicciones sobre porcentajes de asentamiento sobre un sustrato, además de considerar el tipo de sustrato y edad del biofilm, se debe considerar la estación del año en que se sumergen los sustratos, ya que los organismos predominantes de una estación podrían no ser facilitadores o menores inductores del asentamiento larval de ostiones, entre los cuales podrían existir competidores y/o predadores

\section{REFERENCIAS}

Ambrose, W., C. Peterson, H. Summerson \& J. Lin. 1992. Experimental tests of factors affecting recruitment of bay scallops (Argopecten irradians) to spat collectors. Aquaculture, 108:67-86.

Anderson, M. 1996. A chemical cue induces settlement of Sydney rock oyster, Saccostrea commercialis, in the laboratory and in the field. Biol. Bull., 190:350-358.

Anderson, M. \& A. Underwood. 1994. Effects of substratum on the recruitment and development of an intertidal estuarine fouling assemblage. J. Exp. Mar. Biol. Ecol., 184: 217-236.

Bayne, B.J. 1975. The biology of mussel larvae. En: B.L. Bayne (ed.). Marine mussels: their ecology and physiology. Cambridge University Press, New York, pp. 81-115.

Bech, M. 1992. Settling behavior of Chicoreus ramosus (Linneanus, 1758) with notes on sea farming. Phuket Mar. Biol. Center Spec. Publ., 11: 45-52. 
Bonar, D., S. Coon, M. Walch, R. Weiner \& W. Fitt. 1990. Control of oyster settlement and metamorphosis by endogenous and exogenous chemical cues. Bull. Mar. Sci., 46: 484-498.

Bourget, E., J. De Guise \& G. Daigle. 1994. Scales of substratum heterogeneity, structural complexity, and the early establishment of a marine epibenthic community. J. Exp. Mar. Biol. Ecol., 181: 31-51.

Brand, A. \& J. Hoogesteger. 1980. Spat settlement of the scallops Chlamys opercularis (L.) and Pecten maximus (L.) on artificial collectors. J. Mar. Biol. Assoc. U.K., 60: 379-390.

Crisp, D. 1974. Factors influencing the settlement of marine invertebrate larvae. En: P.T Gran \& A.M. Mackie (eds.). Chemoreception in marine organisms. Academic Press, London, pp. 177-265.

Fitt, W., S. Coon, M. Walch, R. Weiner, R. Colwell \& D. Bonar. 1990. Settlement behavior and metamorphosis of oyster larvae (Crassostrea gigas) in response to bacterial supernatans. Mar. Biol., 106: 389-394.

Hadfield, M.G. 1986. Settlement and recruitment of marine invertebrates: a perspective and some proposals. Bull. Mar. Sci., 19(2): 418-425.

Harvey, M., E. Bourget \& R. Ingram. 1995. Experimental evidence of passive accumulation of marine bivalve larvae on filamentous epibenthic structures. Limnol. Oceanogr., 40: 94-104.

Jackson, G.A. 1986. Interaction of physical and biological process in the settlement of planktonic larvae. Bull. Mar. Sci., 39(2): 202-212.

Jacobi, C.M. \& R. Lagevin. 1996. Habitat geometry of benthic susbtrata: effects on arrival and settlement of mobile epifauna. J. Exp. Mar. Biol. Ecol., 206: 39-54.

Jensen, R.A., D.E. Morse, R.L. Petty \& N. Hooker. 1990. Artificial induction of larval metamorphosis by free fatty acids. Mar. Ecol. Prog. Ser., 67: 5571.

Maki, J.S., D. Rittschof, A.R. Schmidt, A. Snyder \& R. Mitchell. 1989. Factors controlling attachment of bryozoan larvae: a comparison of bacterial films and unfilmed surfaces. Biol. Bull., 177: 295-302.

Maki, J.S., D. Rittchof \& R. Mitchell. 1992. Inhibition of larval barnacle attachment to bacterial films: an investigation of physical properties. Microbial Ecol., 23: 97-106.

Marshall, K. 1989. Growth of bacteria on surfacebound substrates: Significance in biofilm development. En: T. Hattari, Y. Yshida, Y. Moruyama, R. Morita \& A. Uchida (eds.). Advances in microbial ecology. Proc. $5^{\text {th }}$ International Symposium on Microbial Ecology, USA. pp. 146-150.

Miron, G., P. Pelletier \& E. Bouget. 1995. Optimizing the design of giant scallop (Placopecten magellanicus) spat collectors: flume experiments. Mar. Biol., 123: 285-291.

Pawlik, J.R. \& M.G. Hadfield. 1990. A symposium on chemical factors that influence the settlement and metamorphosis of marine invertebrate larvae: introduction and perspectives. Bull. Mar. Sci., 46(2): 450-454.

Pearce, C.M. \& E. Bourget. 1996. Settlement of larvae of the giant scallop, Placopecten magellanicus (Gmelin), on various artificial and natural substrata under hatchery-type conditions. Aquaculture, 141: 201-221.

Prieur, D., F. Gail \& S. Corre. 1993. Complex epibiotic bacterial communities on the marine organisms: fouling or interaction? En: R. Guerrero \& C. Pedrós-Alió (eds.). Trends in microbial ecology. Spanish Society for Microbiology, Barcelona, pp. 207-212.

Stokesbury, K. \& J. Himmelman. 1995. Biological and physical variables associated with agreggation of the giant scallop Placopecten magellanicus. Canadian J. Fish. Aquatic Sci., 52: 743-753.

Tamburri, M.N., R. Zimmer-Faust \& M.L. Tamplin. 1992. Natural sources and properties of chemical inducers mediating settlement of oyster larvae: A re-examination. Biol. Bull., 183: 327-338.

Tapia, C., E. Dupré \& G. Bellolio. 1993. Descripción del comportamiento de pedivelígeras de Argopecten purpuratus Lamarck, 1819. Rev. Biol. Mar., 28: 75-84.

Todd, C.D. \& M.J. Keough. 1994. Larval settlement in hard substratum epifaunal assemblages: a manipulative field study of the effects of substratum filming and the presence of incumbents. J. Exp. Mar. Biol. Ecol., 181: 159-187. 
Weiner, R., D. Sledjeski, E. Quintero, S. Coon \& M. Walch. 1993. Periphytic bacteria cue oyster larvae to set on fertile benthic biofilm. En: R. Guerrero y C. Pedrós-Alió (eds.). Trends in microbial ecology. Spanish Society for Microbiology, Barcelona, pp. 217-220.

Wainman, B.C., S. Hincks, N. Kaushik \& G. Mackie. 1996. Biofilm and substrate preference in the dreissenid larvae of lake Erie. Canadian J. Fish. Aquatic Sci., 43: 134-140.

Wolfaart, G.M., J.R. Lawrence, R.D. Robarts, S. Caldwell \& D. Caldwell. 1994. Multicellular organization in a degradative biofilm community. Appl. Environ. Microbiol., 60: 434-446.

Yool, A.J., S.M. Grau, M.G. Hadfield, R.A. Jensen, D.A. Markell \& D.E. Morse. 1986. Excess potasium induces larval metamorphosis in four marine invertebrate species. Biol. Bull., 170: 225 266.

Zimmer-Faust, R.K. \& M.N. Tamburri. 1994. Chemical identity and ecological implications of waterborne, larval settlement cue. Limnol. Oceanogr., 35(5): 1075-1087. 